\title{
Spatial variation of coda wave amplitude in media with a localized heterogeneous region
}

\author{
Kiyoshi Yomogida $^{1 *}$, Rafael Benites ${ }^{2}$, and Rusell Robinson ${ }^{2}$ \\ ${ }^{1}$ Department of Earth and Planetary Systems Science, Faculty of Science, University of Hiroshima, Higashi-Hiroshima 739-8526, Japan \\ ${ }^{2}$ Institute of Geological and Nuclear Sciences, P.O. Box 1320, Wellington, New Zealand \\ (Received July 17, 1997; Revised January 26, 1998; Accepted March 16, 1998)
}

\begin{abstract}
Using an indirect boundary integral method, seismograms are computed for elastic media with localized heterogeneities. Models are two-dimensional homogeneous full spaces (P and SV waves) with many circular cavities as heterogeneities or scatterers. Heterogeneities are localized within a depth range, forming a relatively thin random layer. Seismograms are obtained for receivers at depth $0 \mathrm{~km}$ (surface) and for several focal depths, with sources radiating $\mathrm{S}$ waves isotropically. Seismograms are composed of the direct $\mathrm{S}$ wave and all the possible scattered waves by the heterogeneities, exhibiting late arrivals or coda waves. The coda wave amplitude, or coda energy level, and its duration vary for events with different focal depths. As the focal depth increases and the source gets closer to the layer of localized heterogeneities, the coda level becomes small. When the source is within the heterogeneous layer, however, the coda level becomes larger than for a case of the source either above or below the heterogeneous layer. This local enhancement of coda takes place clearly only in the frequency range for which the scattering is the most effective, that is, when the non-dimensional frequency $k d$ takes values from 2 to 3 , where $k$ is the wavenumber and $d$ is the size of each heterogeneity. Such enhancement of coda is not observed when the density of cavities, or strength of the heterogeneities, is reduced. Coda level becomes locally large for a source within the heterogeneous layer only in the case that the heterogeneities are strong enough to excite multiply scattered waves, as compared with the singly scattered ones. Robinson (1987) studied the temporal variation of coda-duration magnitude relative to event magnitude based on $\mathrm{P}$ and $\mathrm{S}$ wave amplitudes using the seismic network of the Wellington, New Zealand, region. He found that coda-duration magnitude relative to amplitude magnitude decreases with focal depth and becomes large locally for events in the depth range from 65 and $75 \mathrm{~km}$. Our synthetic seismograms explain his results well, implying that there must be a region of localized, strong heterogeneity at depths around $70 \mathrm{~km}$. The effective size of the heterogeneous region may be of about several kilometers because the observation was performed with $1 \mathrm{~Hz}$ seismometers. This localized heterogeneous layer is probably associated with the subducting Pacific plate underneath the Wellington region.
\end{abstract}

\section{Introduction}

Due to the limited quantity and quality of high-frequency seismic observations, small-scale heterogeneities in the earth have been investigated mainly by using stochastic approaches with largely simplified random models. The assumption of stationary random media is essential in almost all the studies on scattering and attenuation of high-frequency seismic waves, that is, the random characteristics are the same everywhere in the media. This stationary random model has been also widely used in theoretical studies since the singlescattering theory of Aki and Chouet (1975), because of its suitability for analytical solutions. The underlying simplification of small-scale heterogeneities in stationary random models has been successful in explaining very complicated features of scattering and attenuation of high-frequency seismic waves.

The recent advance in both seismic observations of high-

*Present address: Division of Earth and Planetary Sciences, Graduate School of Science, Hokkaido University, Sapporo 060-0810, Japan.

Copy right (C) The Society of Geomagnetism and Earth, Planetary and Space Sciences (SGEPSS); The Seismological Society of Japan; The Volcanological Society of Japan; The Geodetic Society of Japan; The Japanese Society for Planetary Sciences. frequency waves and numerical simulations of seismic-wave propagation in complex media is revealing that small-scale heterogeneities are localized in relatively small regions rather than distributed uniformly over large spatial extents. An example is the concentration of highly reflective layers of seismic waves in the lower crust while the upper crust is quite transparent (e.g., Mooney and Brocher, 1987). Another is the concentration of fractures along an active fault zone (e.g., Li et al., 1994). Obara and Sato (1988) identified a region on the top of the subducting Pacific plate at depths around $100 \mathrm{~km}$ underneath the Kanto area, Japan, from late arrival phases of coda. Their observations clearly implied that for high-frequency seismic observations of good quality, the assumption of stationary random media may be too simplistic and the effect of localized heterogeneities more important.

If a random medium is non-stationary, approaches with analytical solutions become extremely difficult. Some of these studies have dealt with media in which the randomness varies with depth (e.g., Hoshiba, 1994; Gusev, 1995). In contrast, the introduction of localized heterogeneities is very simple for numerical approaches. Using the boundary integral method of Benites et al. (1992), Yomogida et al. (1997a) investigated coda $Q$ in two-layer random media, showing that 


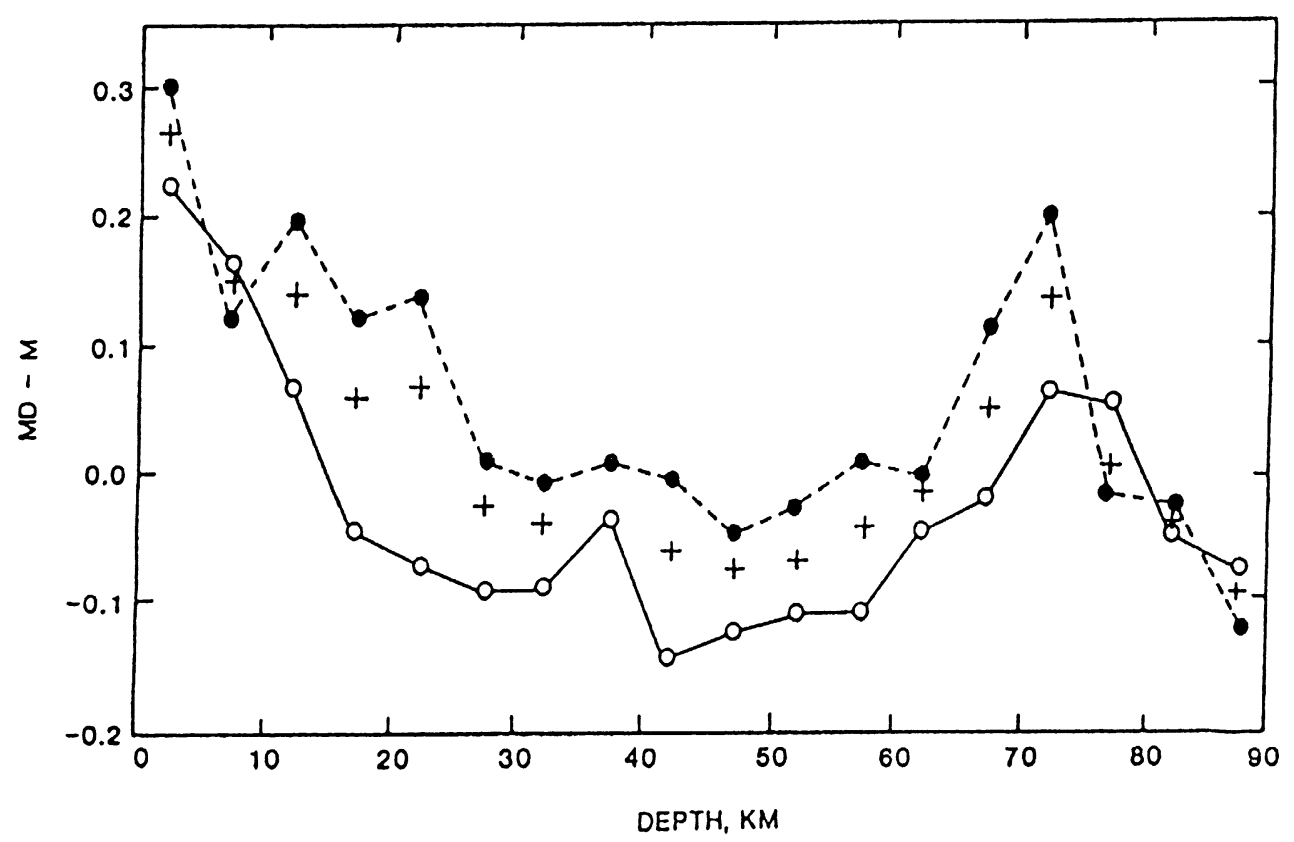

Fig. 1. Variation of coda-duration magnitude $(M D)$ relative to event magnitude $(M)$ as a function of focal depth in the Wellington, New Zealand, region. Solid circles are for the period prior to April 1, 1981, open circles after December 1982 and the averages are represented by crosses. Reproduced from Robinson (1987).

the intrinsic attenuation in the lower crust seems to play the major role in observed coda $Q$ in most cases. Furthermore, Yomogida et al. (1997b) studied the scattering characteristics of media with localized heterogeneities using the method for P-SV waves (two-dimensional) of Benites et al. (1997). They showed that scattered waves vary significantly depending on the type of incident-wave (i.e., $\mathrm{P}$ versus $\mathrm{S}$ waves), scattering angle (i.e., forward versus backward scattering directions) and the size of heterogeneities compared with the considered wavelength.

In this study we investigate scattered waves by localized heterogeneities. In this type of media the scattering should depend strongly on the source location relative to that of heterogeneities, and we shall focus on the variation of coda level with source focal depth in media where heterogeneities are localized within a certain depth range, considering the observation by Robinson (1987).

\section{Evidence for Variation of Coda Strength with Source Depth}

One of the most important applications of coda waves is the so-called "coda-duration magnitude" (Lee et al., 1972), based on the fact that the excitation of coda does not depend on source location, receiver site conditions, and source mechanisms (e.g., Aki, 1969), supported further by systematic data analyses by Aki and Chouet (1975) and Rautian and Khalturin (1978). If the distribution of heterogeneities that excite coda is uniform or the medium is stationary random, the level of coda is proportional to the amplitude of a specific phase so that it can be used to estimate the magnitude of an event. We can replace the level of coda by the duration of coda for seismograms with constant background noise, which is determined by site amplification factor, and a constant value of the temporal decay of coda (coda $Q$ ). Ob- viously, its validity depends strongly on the applicability of simplified media mentioned above, but this scheme appears to measure the magnitude quite reliably as compared with the standard event magnitude, resulting in its wide use (e.g., Herraiz and Espinosa, 1987).

In contrast, the event magnitude is generally determined by the maximum amplitude of $\mathrm{S}$ wave, which strongly depends on various factors such as source mechanism, structure along its propagation path and geological site conditions. Systematic comparisons between coda-duration magnitude and event magnitude have been few because the estimate of event magnitude is biased without highly dense and uniform distribution of stations and careful data analyses. As far as the authors know, Robinson (1987) has been the first to obtain event magnitude of each local event in his study of the Wellington, New Zealand, region by careful estimations of those factors, and to show some systematic discrepancies between those two types of magnitudes.

Robinson (1987) investigated the effect of focal depth on coda duration. Figure 1 shows the variation of the codaduration magnitude relative to the event magnitude as a function of focal depth, and their temporal variations in two periods, before April 1981 and after December 1982. It is observed that coda-duration magnitude systematically decreases with focal depth, down to about $70 \mathrm{~km}$, suggesting certain depth-dependency of the strength of scattering, which in turn depends on the distribution and characteristics of the scatterers. For events with depths around $70 \mathrm{~km}$, the durations of coda become relatively longer. Robinson (1987) speculated that the monotonous variation with focal depth in Fig. 1 might be explained by contamination due to surface waves. In contrast, the peak at $70 \mathrm{~km}$ depth cannot be explained by any simple random media, but by "some struc- 


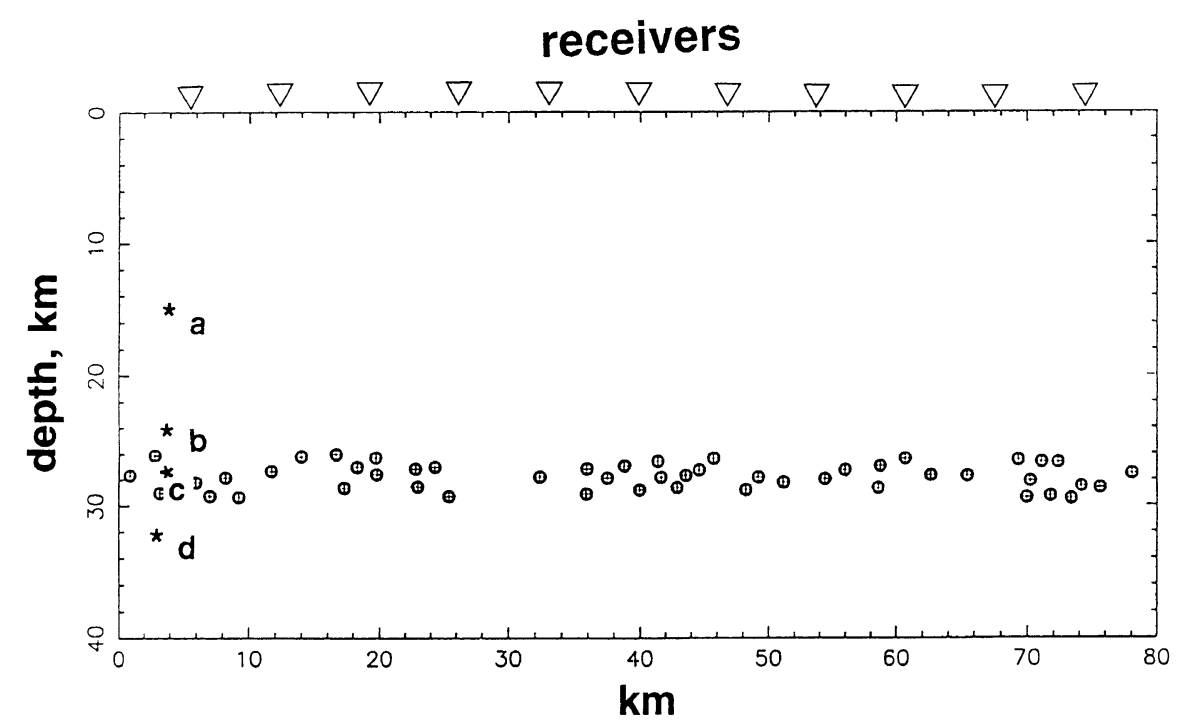

Fig. 2. A two-dimensional homogeneous $\left(6.14 \mathrm{~km} / \mathrm{s}\right.$ and $3.55 \mathrm{~km} / \mathrm{s}$ for P- and S-wave velocities and $2740 \mathrm{~kg} / \mathrm{m}^{3}$ for density) model with 50 circular cavities with the radius of $0.5 \mathrm{~km}$ in the depth range from 25 and $30 \mathrm{~km}$. One-hundred receivers are located at the depth of $0 \mathrm{~km}$. Sources are located with different focal depths: (a) 15, (b) 24 , (c) 27 and (d) $31 \mathrm{~km}$. Each source radiates S waves isotropically.

tural reasons" (Robinson, 1987), which we shall represent as a region of strong heterogeneity.

\section{Coda in Media with Localized Heterogeneities}

In order to explain the variation of coda-duration magnitude with focal depth observed by Robinson (1987), we compute seismograms for media with a distribution of heterogeneities localized at a certain depth, and measure their coda level for several focal depths. In order to focus our attention on the fundamental wave mechanism, we use a simple model, shown in Fig. 2, consisting of a two-dimensional homogeneous full-space model with many circular cavities randomly distributed in the depth range between 25 and $30 \mathrm{~km}$. Receivers are located along the line of $0 \mathrm{~km}$ depth. We choose a source that radiates only $\mathrm{S}$ waves, isotropically, because both event magnitude and coda-duration magnitude are determined by $\mathrm{S}$ waves, and S-to-P scattering is weak (e.g., Yomogida et al., 1997b). Non-isotropic seismic wave radiation due to shear faulting is also not important, at least for coda waves arriving later than twice of the lapse time (Sato et al., 1997).

The use of circular cavities as scatterers has been discussed in detail in previous studies (Benites et al., 1992; Yomogida and Benites, 1995). Although there are no cavities in the earth, the scattering by cavities appears to model observed high-frequency scattered waves quite well, particularly coda waves. The wave mechanism exciting strong scattering seems to be accurately represented by heterogeneities with sharp impedance contrast rather than by media exhibiting continuous, smooth fluctuation of velocity. The characterization of heterogeneities by sharp impedance contrasts is sufficient to explain the dominant features of observed scattered waves, especially coda, discussed in the previous studies (Benites et al., 1992; Yomogida et al., 1997b).

Since all the heterogeneities are far from the receivers, we do not include the effect of the free-surface in our models.
We compute seismograms at one-hundred receivers along the plane of depth equal to zero for events with different focal depths, as shown in Fig. 2. We must emphasize that our model in Fig. 2, a simple crustal layer of 30-40 km thick, is used to perform a comparative study of the dependence of coda amplitude and duration on the depth of the source, without taking into account the deepening of the subduction zone and distribution of seismic activity as in the case of Robinson (1987). We used the boundary integral method of Benites et al. (1997). Figure 3 shows seismograms with focal depths of (a) 15, (b) 24, (c) 27 and (d) $31 \mathrm{~km}$. They are computed for a Ricker wavelet source-time function with the central frequency $1.56 \mathrm{~Hz}$, for both horizontal and vertical components.

When the source is shallow (e.g., case (a)), each phase in the seismograms can be identified from its arrival time. The first simple pulse is the direct $\mathrm{S}$ wave, propagating straight from the source to receivers. Late arrival phases are waves scattered by the cavities. As Yomogida et al. (1997b) pointed out, S-to-P scattered waves can be neglected in most cases. These late arrival phases in Fig. 3 are indeed S-to-S scattered waves, judging from their arrival times and polarization (i.e., vertical versus horizontal components). Some S-to-P phases, represented by "SP", arrive earlier than the direct S wave, but they contribute little to the coda part of the seismograms.

Referring to Fig. 1, which compares coda-duration magnitude with the corresponding event magnitude, in our models the strength of each source is identical; that is, their event magnitudes are all the same. For actual records with background noise, we measure the duration of coda, defining the termination time of coda based on the noise level. This procedure cancels out the site amplification factor so that we can estimate the coda level from the coda duration defined above, which is connected to event magnitude. Since our synthetic seismograms are noise-free, we cannot define the termination time or duration of coda clearly. Indeed, we measured 


\section{a) focal depth: $15 \mathrm{~km}, \mathrm{f}_{\mathrm{c}}=1.56 \mathrm{~Hz}$ b) focal depth: $24 \mathrm{~km}, \mathrm{f}_{\mathrm{c}}=1.56 \mathrm{~Hz}$}
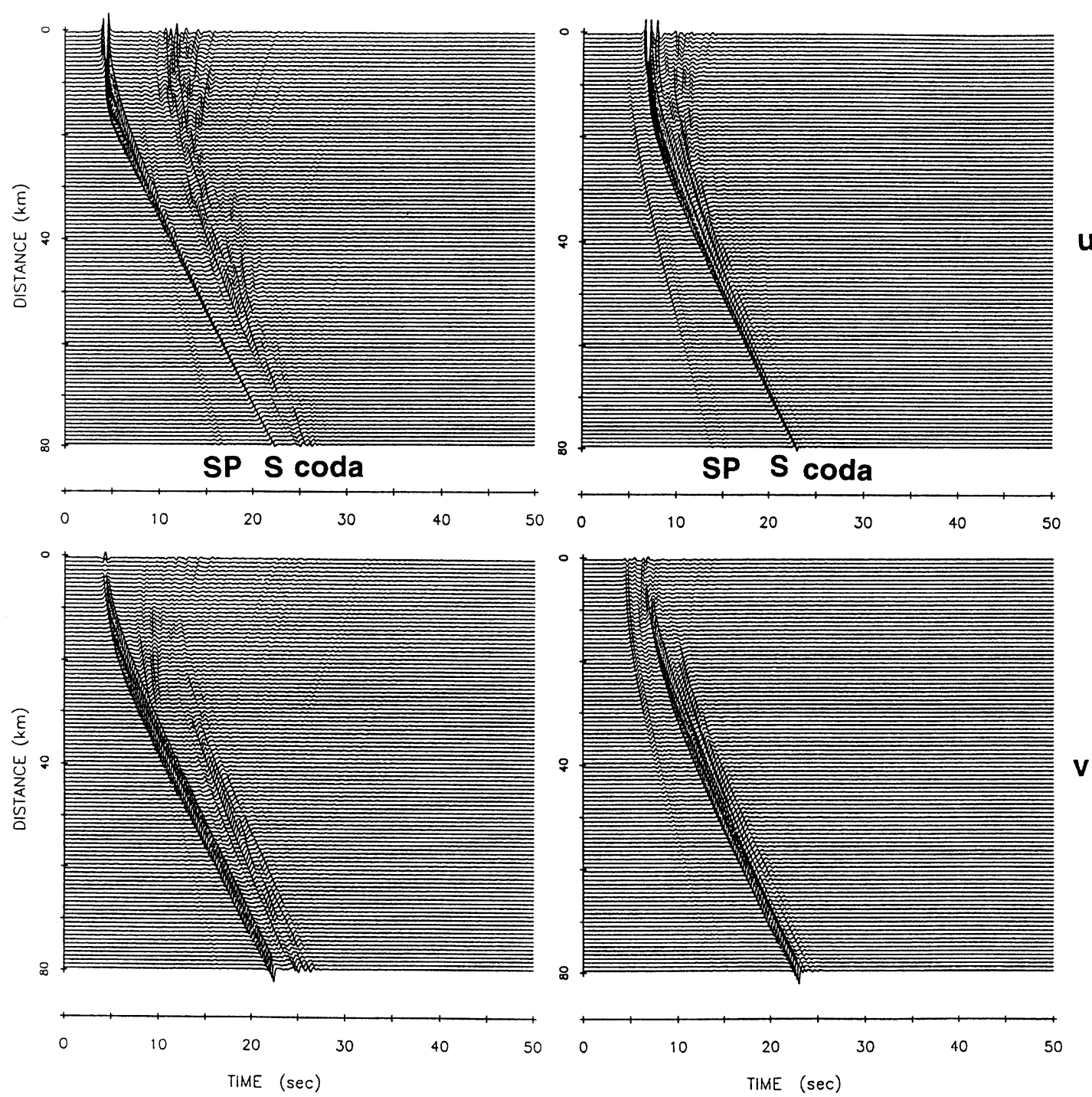

Fig. 3. Seismograms for sources at four different depths $(15,24,27$ and $31 \mathrm{~km})$ in the model of Fig. 2. The top and the bottom of each plot represents horizontal and vertical components. Source-time function is a Ricker wavelet with the central frequency of $1.56 \mathrm{~Hz}$. The direct wave, S-to-P scattered phase, and coda waves (S coda) are represented by "S", "SP", and "coda", respectively.

the coda duration of our synthetics but it strongly depends on how we choose the termination time. The direct use of coda level with a finite time window seems to smooth out variations among seismograms, providing stable values. We therefore replace the coda-duration magnitude in Fig. 1 by the level of coda with our synthetics.

As observed in the synthetic seismograms of Fig. 3 (corresponding to the model of Fig. 2), the amplitude of coda generally decreases as the focal depth increases. When a source is shallow, all the scattered waves are nearly backscattered ones with a wide range of travel distance, producing coda with large amplitude and long duration. In contrast, for a source close to the layer of heterogeneities, early scattered waves, mainly back-scattered ones, interfere with the direct waves while later ones include only a small portion of back-scattered ones, resulting in the suppression of coda generation. As shown in later examples, this tendency holds regardless of the distribution and strength of heterogeneities. The coda amplitude, or coda energy level decreases with focal depth, in agreement with the observation by Robinson (1987).

Another important result in Fig. 3 is the enhancement of the coda level when the source is located within the layer of heterogeneities (i.e., focal depth of $27 \mathrm{~km}$ ), as shown in Fig. 3c. In this case, the scattered waves become locally large, as compared with the case of a source located either above (Fig. 3b) or below (Fig. 3d) the heterogeneous layer. If the source is located within the heterogeneous layer, the excitation of scattered waves is enhanced. The wave mechanisms for such enhancement will be investigated in the following section, but here let us just point out that our model can explain the most significant observational result of Fig. 1. In contrast with the widely used assumption of stationary random media for which the main characteristics of coda, such 
c) focal depth: $27 \mathrm{~km}, \mathrm{f}_{\mathrm{c}}=1.56 \mathrm{~Hz}$ d) focal depth: $31 \mathrm{~km}, \mathrm{f}_{\mathrm{c}}=1.56 \mathrm{~Hz}$
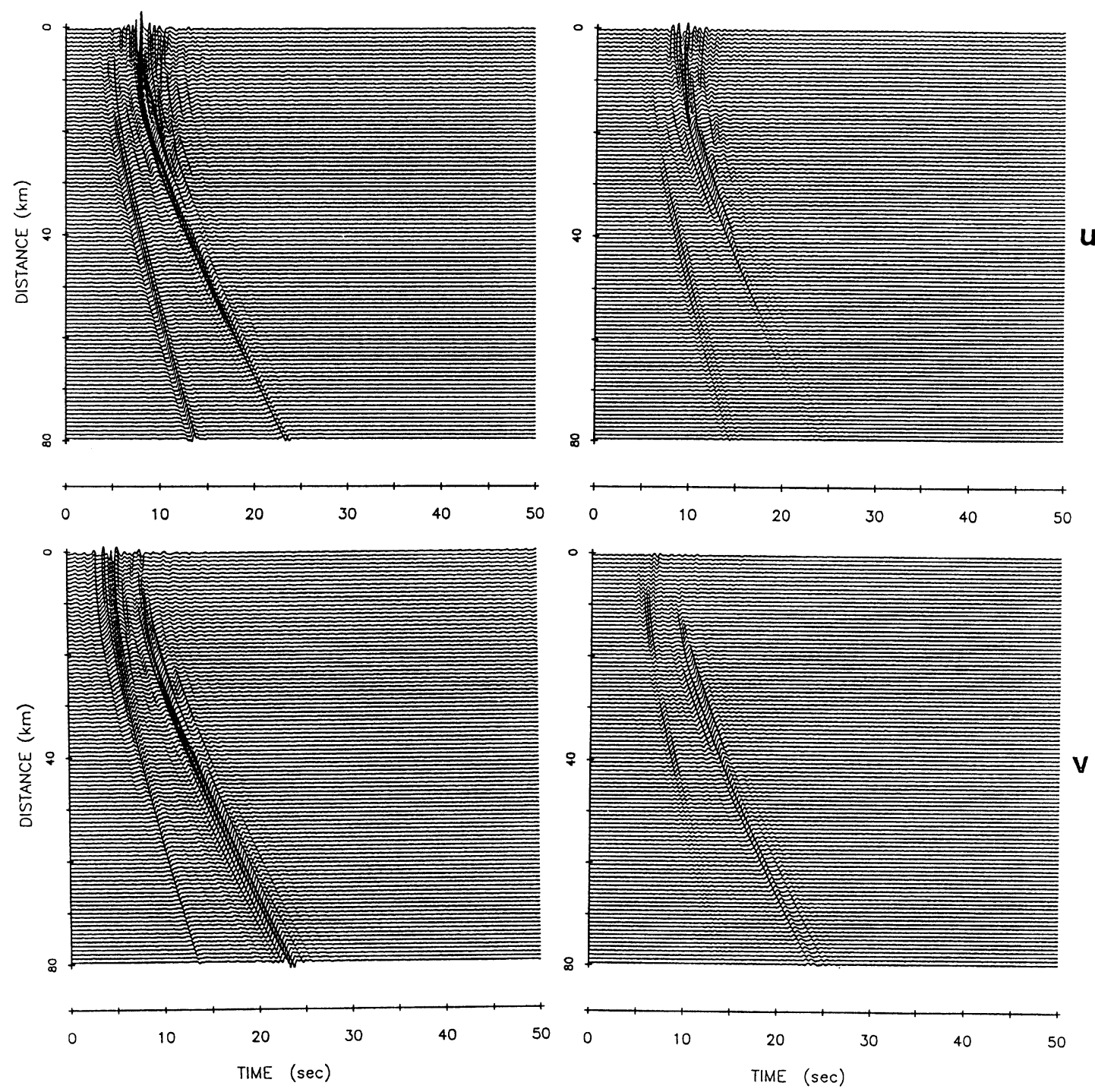

Fig. 3. (continued).

as duration, energy level and decay rate, do not depend on the location of either a source or a receiver (e.g., Aki, 1969), our result supports the suggestion of Robinson (1987) that the relatively large coda-duration magnitude for events with focal depth around $70 \mathrm{~km}$ is due to localized heterogeneities at this depth. In the Wellington region, the Pacific plate is subducting obliquely under the North Island of New Zealand (e.g., Walcott, 1978), and this heterogeneous layer is likely to be related to the subducting slab. Seismic studies (e.g., Chadwick, 1997) in this area have not revealed the existence of any deterministic structures at this depth range, such as a sharp boundary reflector, to explain the anomalous characteristic of coda found in Robinson (1987). Localized heterogeneity represented in a stochastic manner in this study is a more likely candidate.

\section{Role of Multiple Scattering}

From the seismograms in Fig. 3, we have observed that the coda duration increases locally if there is a layer with localized heterogeneities and a source located in it. In this section we discuss the mechanisms of such enhancement.

We first investigate frequency characteristics of coda excitation. Figure 4 shows the seismograms similar to those of Fig. 3 but for a Ricker wavelet of the central frequency $0.78 \mathrm{~Hz}$. Although the coda energy decreases with focal depth and it becomes larger when the source is at $27 \mathrm{~km}$ depth or in the heterogeneous layer (Fig. 4c), the enhancement of coda excitation is not as significant as that for the central frequency $1.56 \mathrm{~Hz}$, suggesting the dependency on frequency.

Scattering of seismic waves depends strongly on frequency or, more precisely, on wavelength relative to the size of the heterogeneities. Both analytical (e.g., Wu, 1982; Sato, 1984) and numerical (e.g., Yomogida and Benites, 1995; Yomogida et al., 1997b) studies have revealed that scattering becomes the strongest when the wavelength of the incident wave, $\lambda$, is comparable to the size of heterogeneities, $d$, that is, for 


\section{a) focal depth: $15 \mathrm{~km}, \mathrm{f}_{\mathrm{c}}=0.78 \mathrm{~Hz}$ b) focal depth: $24 \mathrm{~km}, \mathrm{f}_{\mathrm{c}}=0.78 \mathrm{~Hz}$}
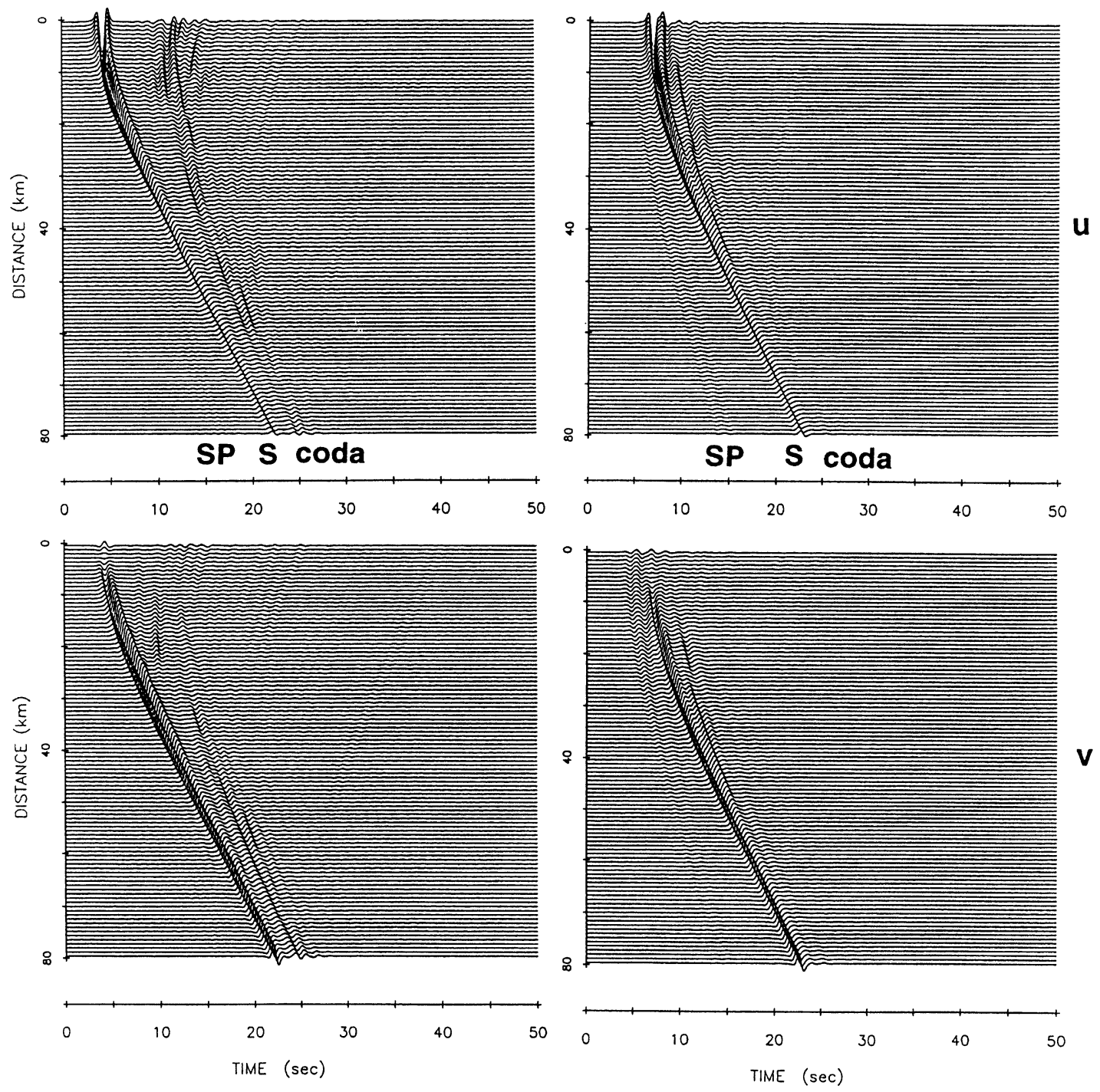

Fig. 4. Same as Fig. 3 except for a Ricker wavelet with the central frequency of $0.78 \mathrm{~Hz}$.

values of the non-dimensional frequency $k d$ between 2 and 3 , with $k$ (wavenumber) $=2 \pi / \lambda$. In the present model, $d$ is the diameter of each cavity and the seismograms in Figs. 3 and 4 correspond to the cases of $k d$ to be 2.76 and 1.38 , respectively. Scattered waves in Fig. 3 are stronger than those in Fig. 4, implying that the enhancement of coda excitation is observed only if the degree of scattering is beyond a certain level.

Changing the strength of scatterers, we compute seismograms with the model shown in Fig. 5. The diameter of each cavity is one-fifth of that of the previous case (Fig. 2) and the number of cavities is four times larger. The fraction of cavities in the depth range from 25 and $30 \mathrm{~km}$ is therefore $4 / 25$ times smaller than that of the model in Fig. 2. Seismograms for this model are shown in Fig. 6 with four different focal depths. The central frequency of a Ricker wavelet is $1.56 \mathrm{~Hz}$.

The overall features of the seismograms are quite simi- lar to those of the previous model, although the coda level is generally smaller. The coda level decreases with focal depth, which agrees with the previous result, but a significant difference is noticed when the source is located within the heterogeneous layer (Fig. 6c). Unlike the previous seismograms, the level or duration of coda for the focal depth of $27 \mathrm{~km}$ is clearly smaller than that for the focal depth of $24 \mathrm{~km}$, just above the heterogeneous layer (Fig. 6b). For the model with weak heterogeneities (Fig. 5), the excitation of coda is not enhanced for a source located inside the region of localized heterogeneities.

Yomogida and Benites (1995) estimated the strength of multiple scattering relative to single scattering by the comparison of complete seismograms with seismograms composed only of singly scattered waves. From their results, the model in Fig. 2 has a fraction of cavities large enough to excite multiply scattered waves as compared with singly scattered ones, particularly in the frequency range for which 
c) focal depth: $27 \mathrm{~km}, \mathrm{f}_{\mathrm{c}}=0.78 \mathrm{~Hz}$
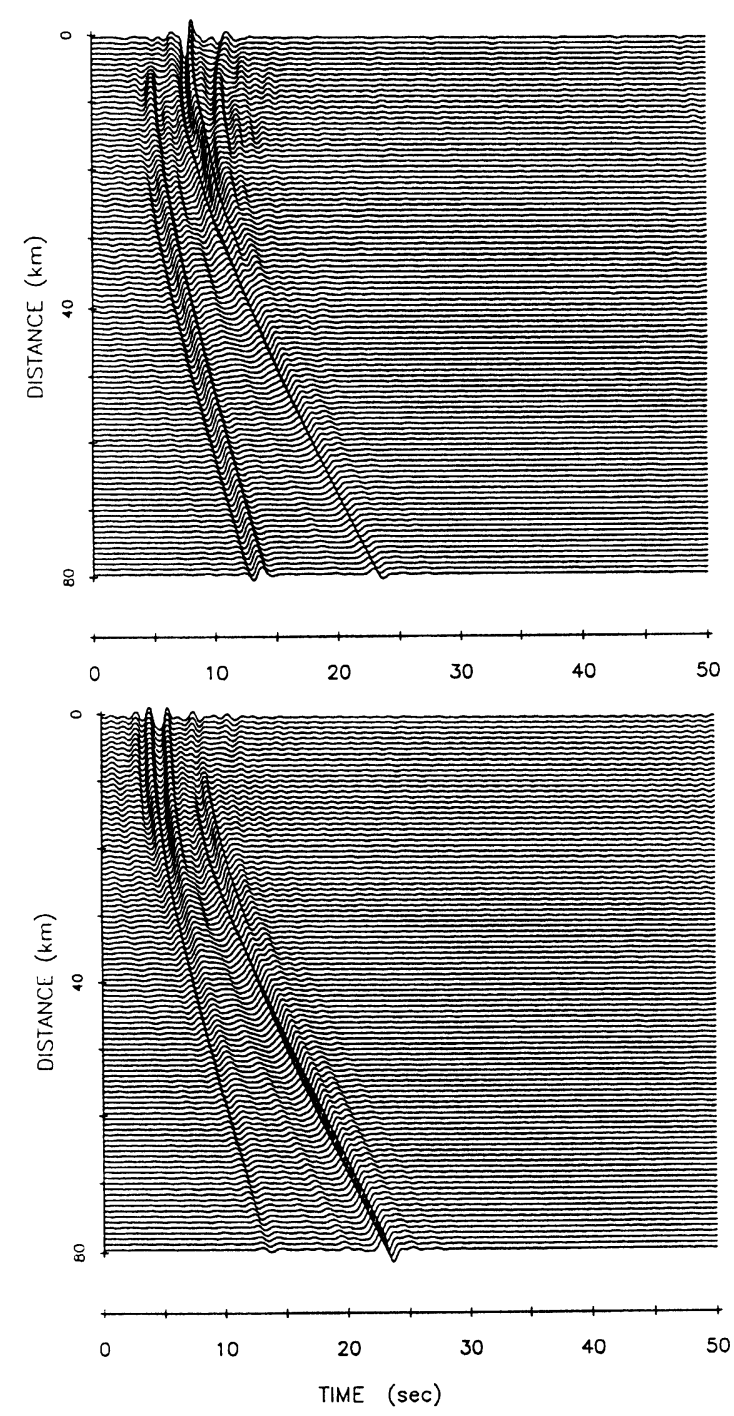

d) focal depth: $31 \mathrm{~km}, \mathrm{f}_{\mathrm{c}}=0.78 \mathrm{~Hz}$
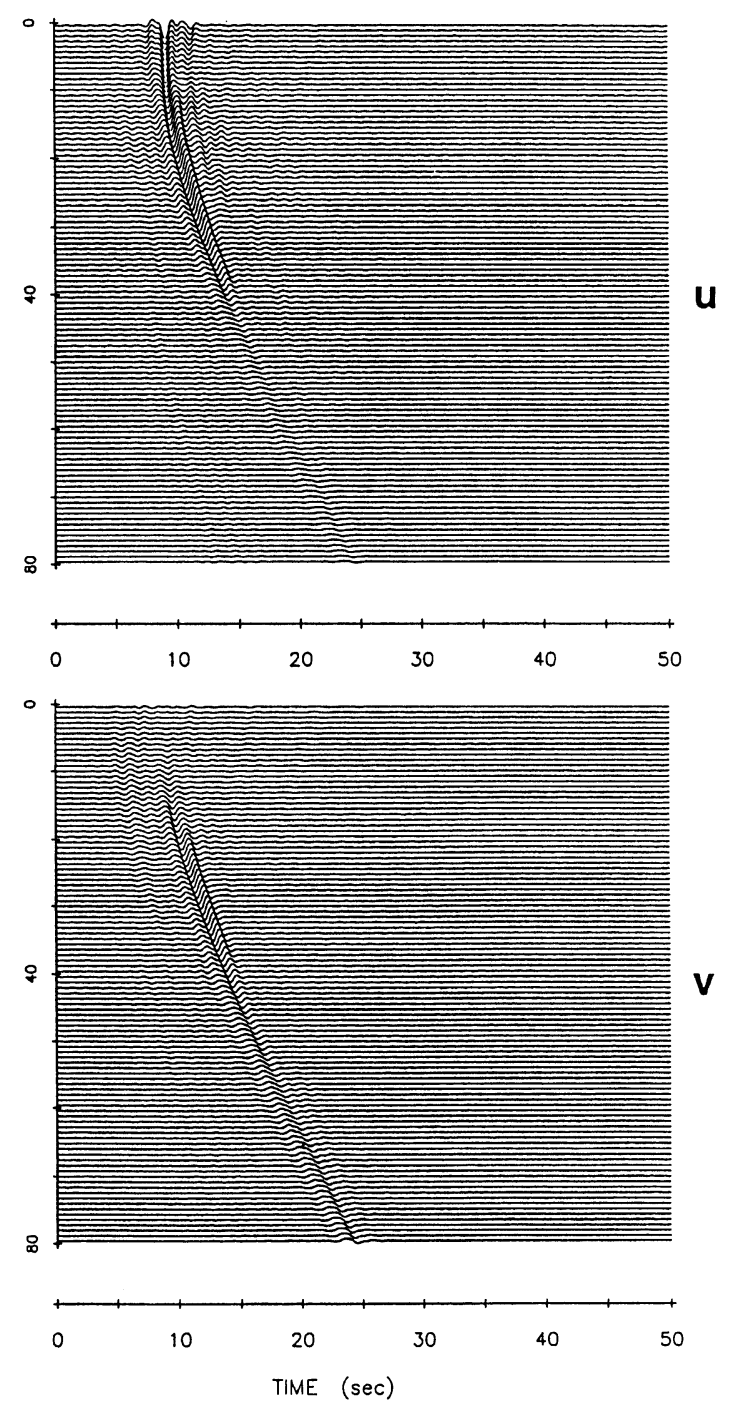

Fig. 4. (continued).

the attenuation due to scattering peaks, that is, for $k d=2$ to 3 , as mentioned before. On the other hand, the concentration of cavities of the model in Fig. 5 is so small that the effect of multiple scattering is minor in any frequency range.

We summarize in Fig. 7 the spatial variations of coda level with focal depth shown in our synthetic seismograms of Figs. 3, 4 and 6. We intend to compare this plot with Fig. 1, although there remain various factors that have not been taken into consideration in the models. We define "coda level" in this figure as the sum of power spectra of horizontal and vertical components within a time window for all the seismograms. The time window is 10 seconds after the maximum amplitude of coda (i.e., excluding the direct $\mathrm{S}$ wave). Even using different time-window lengths, the overall feature of this diagram does not change significantly. The reason why we use coda level instead of coda duration is given in the previous chapter.

The coda level decreases with focal depth in all cases, agreeing with the observational result of Fig. 1. The coda level becomes large locally for focal depths between 25 and $30 \mathrm{~km}$, or within the heterogeneous layer, only in the case that the heterogeneities are strong and the frequency is $1.56 \mathrm{~Hz}$ or $k d$ is 2.76 . In other words, the coda level becomes large for a source located within the layer of localized heterogeneities when multiple scattering is dominant compared with single scattering. From the observational result in Fig. 1 we cannot estimate the size and elastic characteristics of the heterogeneities likely localized in the depth range about $70 \mathrm{~km}$. Nevertheless, we can conclude that the heterogeneities at this depth must be strong enough to excite dominant multiply scattered waves, and the size of heterogeneities must be comparable to the wavelength of the seismograms used for Fig. 1 (1 Hz vertical seismometers).

\section{Conclusions}

Using synthetic seismograms computed with the boundary integral method of Benites et al. (1997), we investigated the 


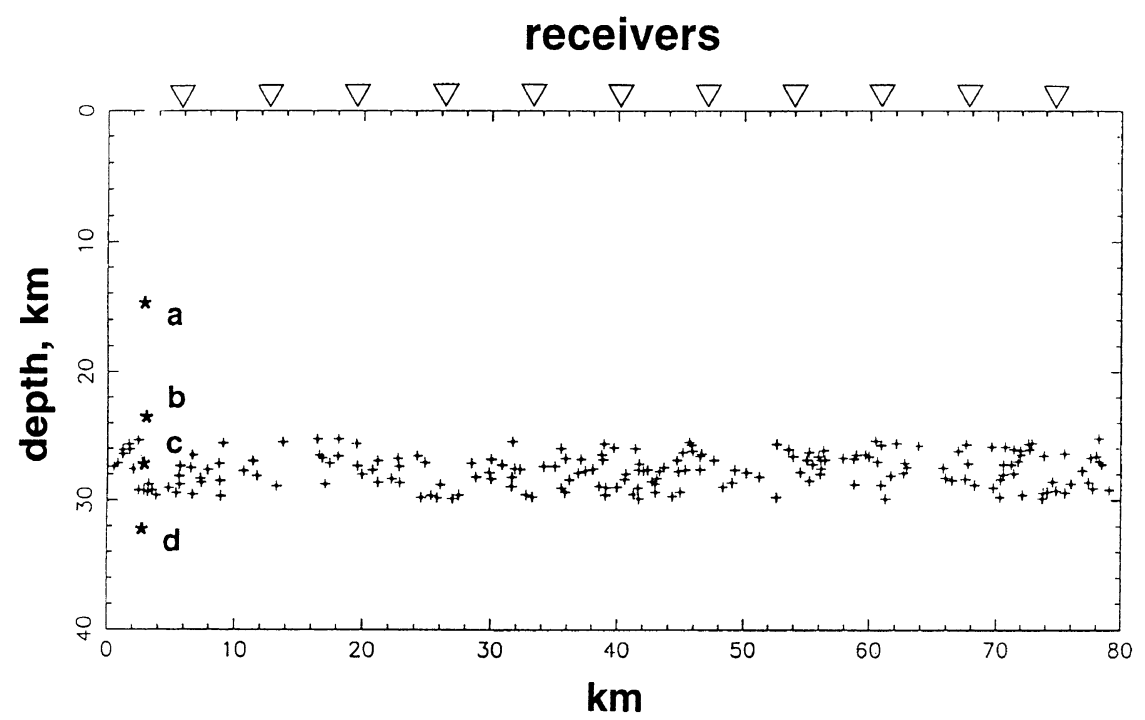

Fig. 5. A two-dimensional homogeneous model with 200 circular cavities with the radius of $0.1 \mathrm{~km}$ in the depth range of 25 and $30 \mathrm{~km}$.
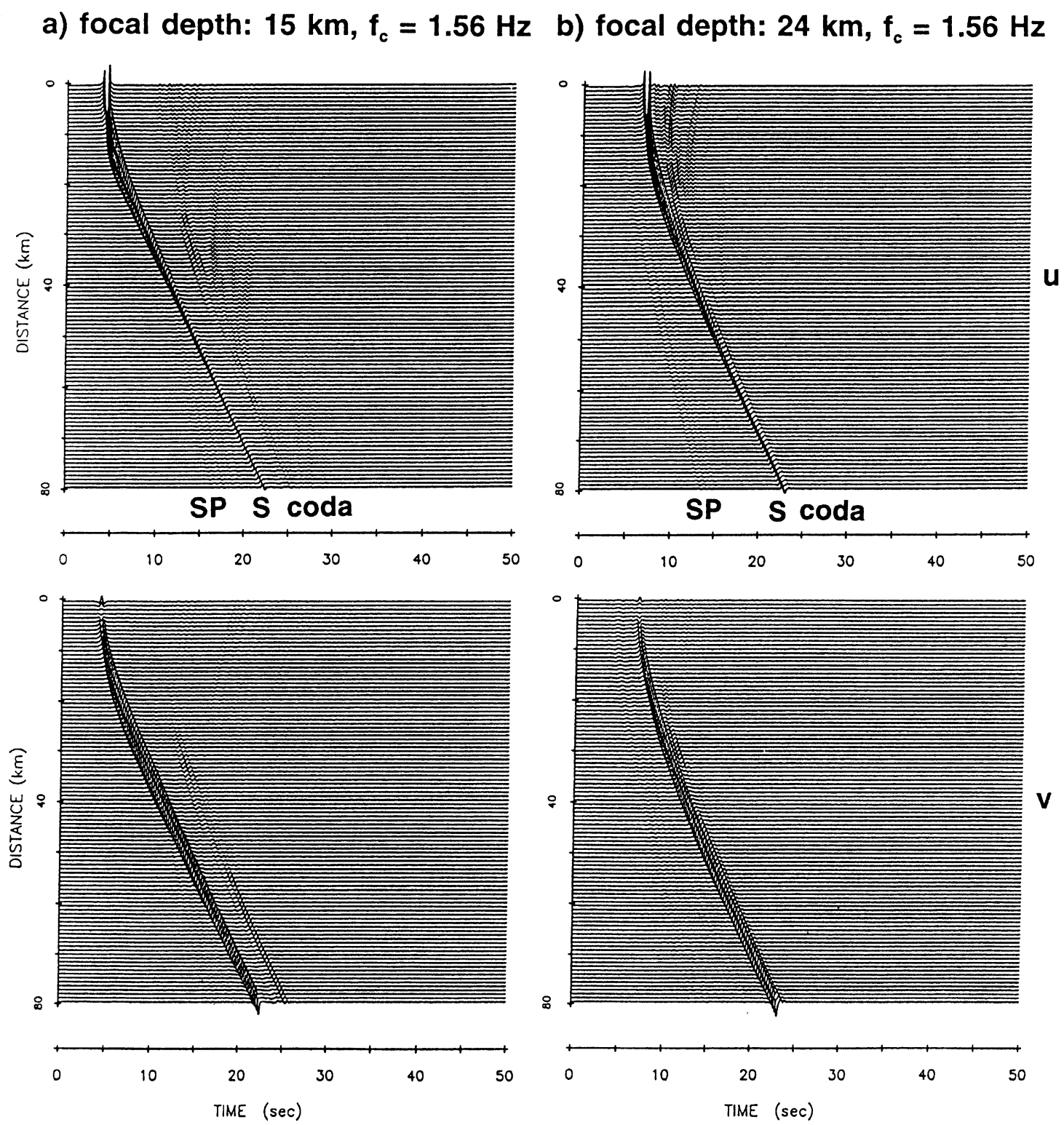

Fig. 6. Same as Fig. 3 except for the model of Fig. 5. 
c) focal depth: $27 \mathrm{~km}, \mathrm{f}_{\mathrm{c}}=1.56 \mathrm{~Hz}$
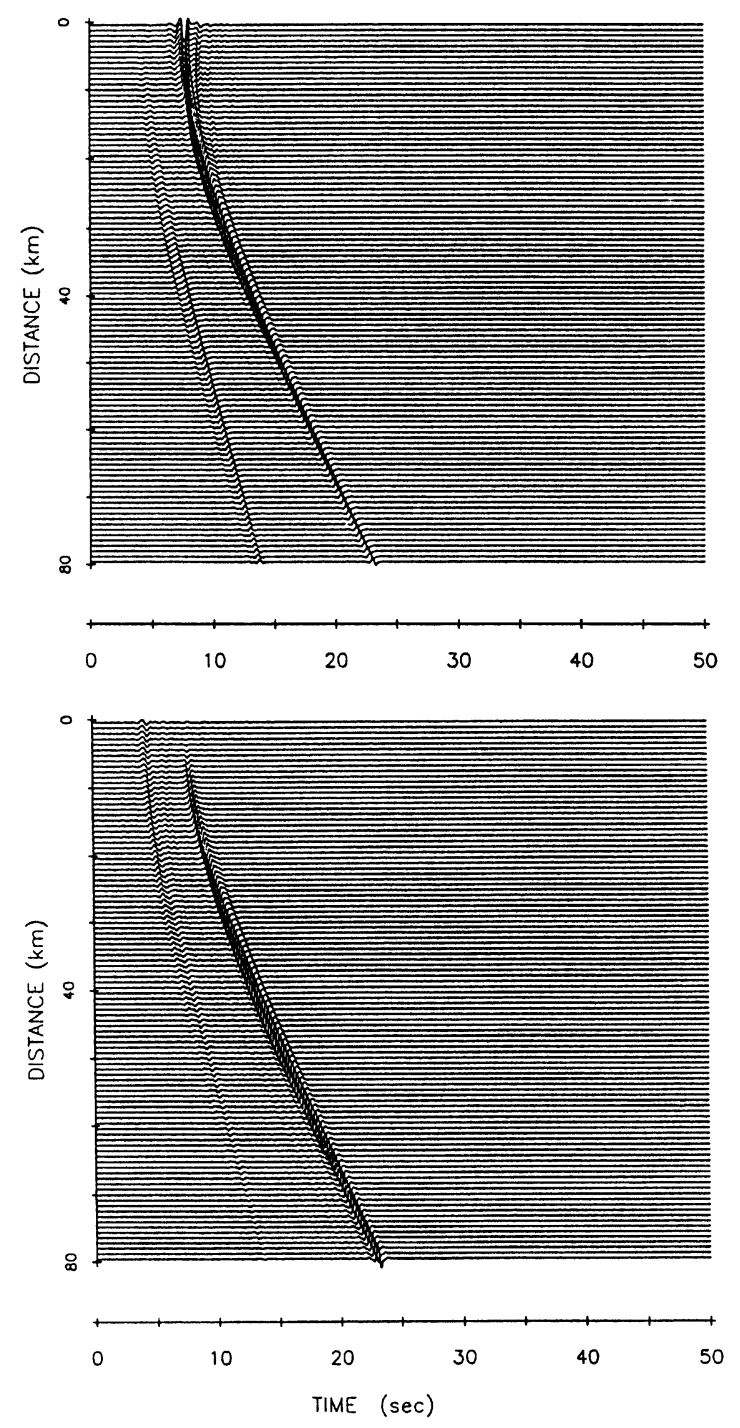

d) focal depth: $31 \mathrm{~km}, \mathrm{f}_{\mathrm{c}}=1.56 \mathrm{~Hz}$
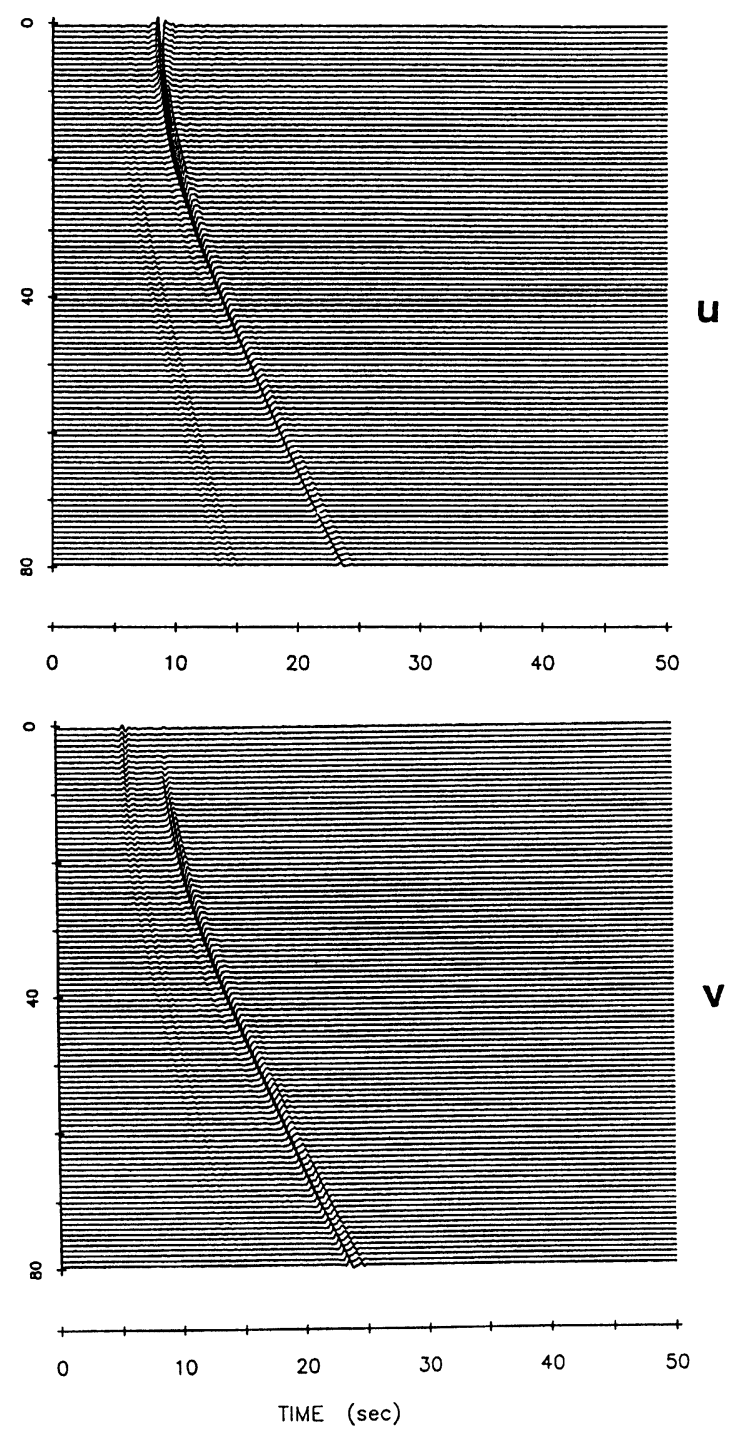

Fig. 6. (continued).

spatial variation of seismic coda waves with focal depth. For media with localized heterogeneities, the coda level, taken as the total duration of coda in seismic observations (Lee et al., 1972), is affected by the source location relative to the heterogeneous region. If the heterogeneities are concentrated in a certain depth range, the coda level at receivers on the surface decreases as focal depth increases because the waves scattered by the heterogeneities interfere the direct wave severely with small amounts of back scattering. When the source is below the heterogeneous layer, the coda level decreases further, most likely because the receivers are located in the direction of forward scattering where coda is generally weak (Yomogida et al., 1997b).

If the heterogeneities are strong enough to excite multiply scattered waves (size comparable to the wavelength), coda increases locally when the source is located within the region of heterogeneities. If not, the enhancement of coda excitation is not observed, in any case. This result explains the spatial variation of coda-duration magnitude relative to the event magnitude, with focal depth in the Wellington, New Zealand, region, studied by Robinson (1987). The systematic increase of coda-duration magnitude for events in the depth range from 65 to $75 \mathrm{~km}$ implies that there must be a region at such a depth range where heterogeneities are sufficiently dense to excite strong multiply scattered waves. Judging from the tectonic setting, this region may be associated with that part of the subducting Pacific plate in which phase changes (basalt to eclogite) are taking place.

From the numerical point of view, it would be possible to estimate the elastic and geometrical parameters that describe the nature of the localized heterogeneities. The observational information contained in Fig. 1 is, however, too little. Coda-duration magnitude was developed for practical purposes (i.e., an alternative to event magnitude based on $\mathrm{P}$ and $\mathrm{S}$ amplitudes) but is not appropriate to describe scattered waves quantitatively. Using the waveforms of scattered 


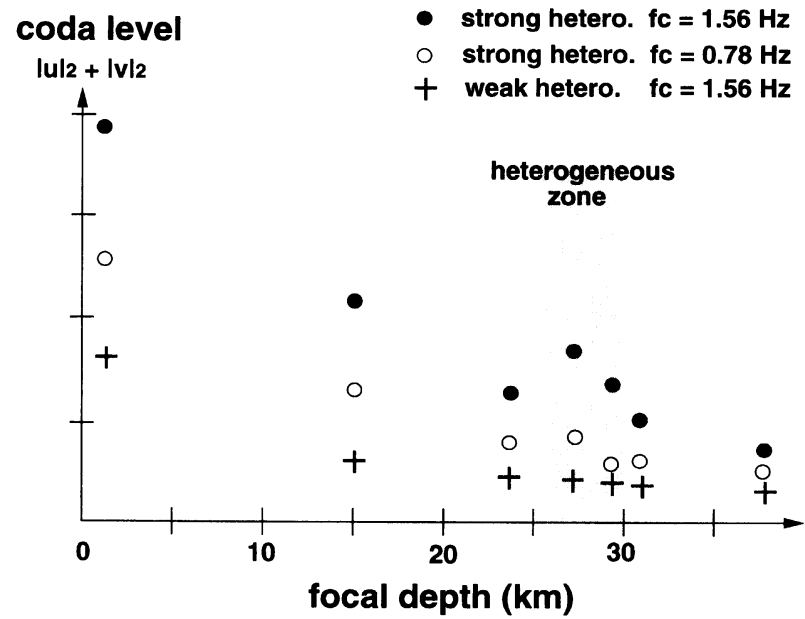

Fig. 7. Variation of coda level with different focal depths for seismograms of Fig. 3 (solid circles), Fig. 4 (open circles) and Fig. 6 (crosses). Coda level is defined as the sum of horizontal- and vertical-component power spectra with the time window of 10 seconds beginning at the maximum of coda.

waves, systematic observations of spatial variations with focal depth are required in order to further quantify the localized heterogeneities. Another simple but important observation suggested for future studies is how the spatial variation in Fig. 1 depends on frequency. If we could obtain such a result for several frequency ranges and compare them, then the size of the localized heterogeneities could be precisely estimated. Broadband seismic observations will comply with such a goal.

Reliable evidence of non-stationary randomness (i.e., heterogeneities not distributed uniformly) is just starting to be obtained in high-frequency seismic observations. Dense seismic arrays as well as broadband networks have been deployed in many seismically active areas over the world. The present study shows that both observational and numerical studies are able to address the nature of localized, small-scale heterogeneities in the earth quantitatively. The observation discussed in this study (Fig. 1) suggests a spatial variation of heterogeneities with depth. In addition, volcanic regions or active-fault zones are considered to have strong localized heterogeneities even in the horizontal direction. Future observations of such heterogeneities will be also simulated by the present approach.

Acknowledgments. Computations were performed mainly at the computer facilities of the Earthquake Research Institute (University of Tokyo) and the Institute for Numerical Simulations and Applied Mathematics (University of Hiroshima). Most of the present study was done while the first author (K.Y.) was visiting the Institute of Geological and Nuclear Sciences of New Zealand by its support as well as a grant from the Bilateral Research Activities Programme of the Ministry for Science and Technology of New Zealand, for which the help of Alan Hull is greatly appreciated.

\section{References}

Aki, K., Analysis of seismic coda of local earthquakes as scattered waves, J. Geophys. Res., 74, 615-631, 1969.

Aki, K. and B. Chouet, Origin of coda waves: Source, attenuation, and scattering effects, J. Geophys. Res., 80, 3322-3342, 1975.

Benites, R., K. Aki, and K. Yomogida, Multiple scattering of SH waves in 2-D media with many cavities, Pure Appl. Geophys., 138, 353-390, 1992.

Benites, R., K. Yomogida, P. M. Roberts, and M. Fehler, Scattering of elastic waves in 2-D composite media I: Theory and test, Phys. Earth Planet. Inter., 104, 161-173, 1997.

Chadwick, M. C., The 1991 Hikurangi margin seismic refraction profile, Ph.D. Thesis, Victoria University of Wellington, Wellington, New Zealand, pp. 234, 1997.

Gusev, A. A., Vertical profile of turbidity and coda $Q$, Geophys. J. Int., 123 665-672, 1995 .

Herraiz, M. and A. F. Espinosa, Coda waves: A review, Pure Appl. Geophys., 125, 499-577, 1987.

Hoshiba, M., Simulation of coda wave envelope in depth dependent scattering, Geophys. Res. Lett., 21, 2853-2856, 1994.

Lee, W. H. K., R. E. Bennett, and K. L. Meagher, A method of estimated magnitude of local earthquakes from signal duration, U.S. Geol. Surv. Open File Report, 28, 1972.

Li, Y.-G., K. Aki, D. Adams, and A. Hasemi, Seismic guided waves trapped in the fault zone of the Landers, California, earthquake of 1992, J. Geophys. Res., 99, 11705-11722, 1994.

Mooney, W. D. and T. M. Brocher, Coincident seismic reflection/refraction studies of the continental lithosphere: A global review, Rev. Geophys., 25, 723-742, 1987.

Obara, K. and H. Sato, Existence of an S wave reflector near the upper plane of the double seismic zone beneath the southern Kanto district, Japan, $J$. Geophys. Res., 93, 15037-15045, 1988.

Rautian, T. G. and V. I. Khalturin, The use of the coda for determination of the earthquake source spectrum, Bull. Seismol. Soc. Amer., 68, 923-948, 1978

Robinson, R., Temporal variations in coda duration of local earthquakes in the Wellington region, New Zealand, Pure Appl. Geophys., 125, 579-596, 1987.

Sato, H., Attenuation of envelope formation of three-component seismograms of small local earthquakes in randomly inhomogeneous lithosphere, J. Geophys. Res., 89, 1221-1241, 1984.

Sato, H., H. Nakahara, and M. Ohtake, Synthesis of scattered energy density for non-spherical radiation from a point shear-dislocation source based on the radiative transfer theory, Phys. Earth Planet. Inter., 104, 1-13, 1997.

Walcott, R. I., Geodetic strains and large earthquakes in the axial tectonic belt of North Island, New Zealand, J. Geophys. Res., 83, 4419-4429, 1978.

Wu, R. S., Attenuation of short period seismic waves due to scattering, Geophys. Res. Lett., 9, 9-12, 1982.

Yomogida, K. and R. Benites, Relation between direct wave $Q$ and coda $Q$ A numerical approach, Geophys. J. Int., 123, 471-483, 1995.

Yomogida, K., K. Aki, and R. Benites, Coda $Q$ in two-layer random media, Geophys. J. Int., 128, 425-433, 1997a.

Yomogida, K., R. Benites, P. M. Roberts, and M. Fehler, Scattering of elastic waves in 2-D composite media II: Waveforms and spectra, Phys. Earth Planet. Inter., 104, 175-192, $1997 \mathrm{~b}$.

K. Yomogida (e-mail: yomo@geol.sci.hiroshima-u.ac.jp), R. Benites (email: R.Benites@gns.cri.nz), and R. Robinson (e-mail: R.Robinson@gns. cri.nz) 